Research article

\title{
New record of Cyanoprokaryotes from West Bengal in Maldah district
}

\author{
Pratibha Gupta \\ Botanical Survey of India, Ministry of Environment Forests \& Climate Change, Government of India, \\ AJCBIBG, CNH Building, Botanic Garden, Howrah-711103, India \\ *Corresponding Author: drpratibha2011@ rediffmail.com \\ [Accepted: 22 November 2017]

\begin{abstract}
Systematic survey and collection of Cyanoprokaryotes were carried out from different water bodies of Maldah District, West Bengal. During survey samples were sampled from 55 different water bodies of this area comprising 05 sites in rivers, 32 bils, 07 dighis, 02 Jheels, 09 ponds for which surveyed all administrative blocks of Maldah District namely Ratua I, Ratua II, Harishchandrapur I, Harishchandrapur II, Chanchal I, Chanchal II, Manikchak, Gazol, Habibpur, Bamangola, Old Maldah, English Bazar and Kaliachak. During the study, altogether 22 genera and 105 species (comprising 93 species, 09 varieties and 03 forms) were identified from different types of water bodies of Maldah District. Out of these 105, 27 species have been recorded from West Bengal. These species have been described here along with nomenclature and distribution.

Keywords: Cyanoprokaryotes - New Record - Maldah District - West Bengal.
\end{abstract}

[Cite as: Gupta P (2017) New record of Cyanoprokaryotes from West Bengal in Maldah district. Tropical Plant Research 4(3): 421-432]

\section{INTRODUCTION}

Maldah district is one of important district among 19 districts of West Bengal. The major river the Ganges flows along south-western boundary of the district followed by another major rivers like Mahananda, Fulhar and Kalindri. All above rivers originated basically from the Himalayan or sub-Himalayan region and flow Southerly direction whereas some seasonal rivers like Tangoan, Punarbhaba, Pagla and Bhagirathi are also flowing through district territory. Thus, in terms of duration and frequency of flood and its recurrences is concerned, Maldah district is defined as a chronically flood-affected area. Swamps (bils) are extending along the right bank of Mahananda from Kalindri. The practice of aquaculture, cultivation of rice and Makhana (Eurayle ferox Salisb.) are other aspects on which socio-economic condition and livelihood of the district depend. To meet the requirements, a huge number of big and small size 'dighi' and 'ponds' depending upon land area available to individuals are made by them. Such water bodies often get eutrophicated by micro and macrophytes as a result of contaminants from various non-point and point sources like runoff of agriculture fields in which huge quantity of fertilizers and pesticides is used and civic as well as industrial pollutants respectively. Among the aquatic flora, diversity and distribution of microscopic flora is much greater having both beneficial and harmful properties. Therefore, exploration of all forms of different community is warranted which is not possible to be studied by any individual. Thus, during the study, particularly concentrated on Cyanoprokaryota for proper exploration of the unexplored area i.e. Maldah District of the State of West Bengal.

The ancient Cyanoprokaryota likely produced much of oxygen in the Earth's atmosphere, as they dominantly metabolise and fix carbon in the form of sugars by using carbon dioxide. Increase in concentration of oxygen on the earth crust, recorded during 2.4 billion years ago considered as an architect of earth's atmosphere as they are "nature's first and foundational mother and father for causing photosynthesis", entail to form pure ecological niche on our planet and precisely stands as the founder of the aquatic food-chain. As per findings, they have been distributed all over land and water system often in such an environment where there is no other vegetation possibly due to their adaptive capability to extreme adverse environmental conditions with respect to different environmental factors like temperature, $\mathrm{pH}$, salinity, availability of nutrients, pollution load, etc (Sridhar et al. 2006, Bajpai et al. 2013). Their occurrence even in the wide range of ecologically stress conditions and extreme 
habitats proves that they are very tolerant and thus placed in a unique group of micro-organisms. They occur in a fresh-water ecosystem like bils, dighis, lakes, rivers, wetlands, waterfall, etc. and marine water system like salt marshes and pans, estuaries, brackish waters and ocean. Besides, it also occur on inter-tidal rocks, snow and even in cold lakes underneath $5 \mathrm{~m}$ of ice pack as well as in thermal springs, soil, acid bogs and alkaline soils or more, in sub-aerial habitats like tree trunks, moist walls and all other objects which remain moisten and get solar light from any angle even in a short span of time.

Enormous work on quantitative and qualitative along with taxonomic studies, indicator of water pollution / environmental assessments and R \& D studies using selected species have been carried out in different parts of India (Mohan et al. 2007, Bajpai et al. 2013, Malathi et al. 2014, Srivastava et al. 2014, Halder 2015, Jitendra \& Anand 2016, Patel et al. 2017). So far, out of 19 districts of West Bengal such studies have been carried out only in some of the Districts and surrounding area comprising Bankura (Sinha \& Mukherjee 1984, Mukherjee 1986), Burdwan (Gupta \& Sen 1978, Chatterjee \& Choudhury 1980, Chatterjee \& Chatterjee 1983), Hooghly (Gupta \& Sen 1987, Banerjee 1998, Sikdar \& Keshri 2014), Howrah (Mukhopadhyay \& Chatterjee 1981, Sabata \& Nayer 1992, Sen \& Gupta 1993), Kolkata (Biswas 1925, 1926, Santra 1987, Mitra \& Gupta 1994, Banerjee 1997, Sen 2006), Midnapur (Pal \& Santra 1985), Murshidabad (Pal \& Santra 1982, 1984, Pal et al. 1986), 24-Paraganas (Mukhopadhyay \& Chatterjee 1981, Maity \& Santra 1985, Singh et al. 2001, Naskar et al. 2008), Sunderban (Pal et al. 1988, Banerjee \& Santra 2001) and in continuation for further exploration in remaining Districts of the West Bengal, Maldah district was considered first keeping in view to explore Cyanoprokaryotes forms taxonomically.

\section{MATERIALS AND METHODS}

Maldah district is situated in West Bengal of north-east India and lies between $24^{\circ} 41^{\prime} 20^{\prime \prime}$ and $25^{\circ} 32^{\prime} 08^{\prime \prime}$ North Latitude and $87^{\circ} 45^{\prime} 50^{\prime \prime}$ and $88^{\circ} 28^{\prime} 10^{\prime \prime}$ East Longitude, extends over $3733.17 \mathrm{~km}^{2}$ with total population 32,90,468 as per Census, 2001 (BAES 2004) and English Bazar is the District Administrative Headquarter. The district is bounded to its south by the district of Murshidabad across the river Ganga, by Rajshahi district of Bangladesh and Dakshin Dinajpur district to its east and north-east, by Uttar Dinajpur district to its direct north and by the Purnea of Bihar to its direct west and by Sahibganj of State of Jharkhand across the Ganga to the south-west (Fig. 1).

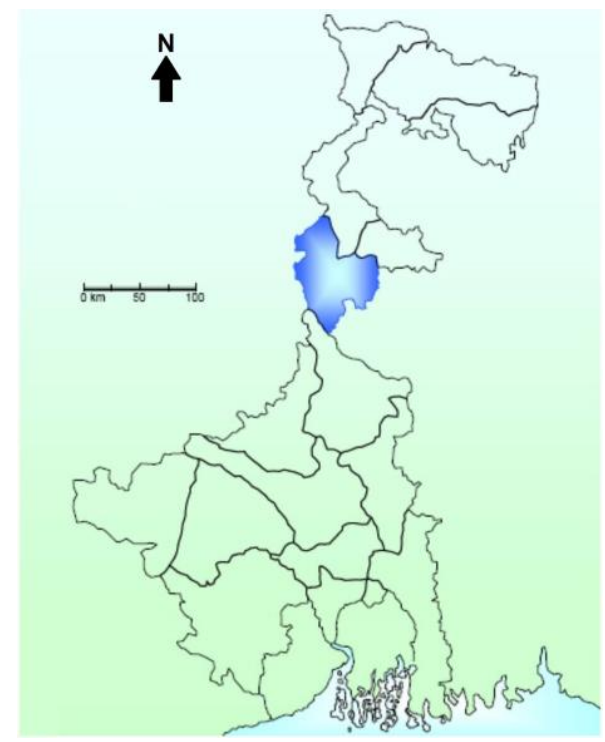

Figure 1. Location of the study site.

During the survey all administrative blocks of Maldah district namely Ratua I, Ratua II, Harishchandrapur I, Harishchandrapur II, Chanchal I, Chanchal II, Manikchak, Gazol, Habibpur, Bamangola, Old Maldah, English Bazar and Kaliachak were visited and samples containing cyanophycean forms were sampled from 55 water bodies comprising 5 sites in rivers like Kalindri (Bhaluka), Fulhar (Sankatala Ghat), Ganga (Mahadhap and Gopalpur) and Mahananda (Alal Bridge); 32 bils (Amkhaki, Liltua, Barabilla, Singera, Ghogha, Chakla, Bochamari, Ashi Dob, Makaiya, Hazartakia, Janipukur, Adhsoi, Manna, Dekul, Meetna, Singsar, Sanak, Singer, Kendha, Kuchla, Bhatia, Chand, Tarkeshwar, Nawabganj, Balotuli, Jalsukha, Laxmipur, Chattara, Garhal, Adhsoi, Pulintola and Madhaipur); 7 dighis (Raikhan, Thinnagar, Kalua, Paradhala, Sukan, Bara and Chota www.tropicalplantresearch.com 
Sagar); 2 Jheels (Bochahi and Mahadhap) and 9 ponds / Pukurs (Bhadobartola, Damua, Shivrampalli, Rohni, Meenatulla, Jorkuppa, Kuppa, Samda and Salamidarwaza).

Samples were sampled randomly towing Phytoplankton net to a distance of 1.0-5.0 m depending upon the depth of water bodies. The samples were preserved in $15.0 \mathrm{ml}$ screw cap Borosil glass specimen vials to avoid any chemical reaction. To take samples from another water bodies, Phytoplankton net was thoroughly washed with clean water after collection of each sample. Samples were preserved by adding 2-3 drops of 4\% Formalin solution. Specimens were observed under Leica DM 2500 Microscope and photomicrograph of each specimen was taken by DFC 500 digital camera with annotation using Leica QWin V 3.2 Image Processing and Analysis Software and Leica Application Suit V4. Specimens were identified by consulting standard books, monograph (Geitler 1932, Tiffany \& Britton 1952, Desikachary 1959, Prescott 1982, Anand 1989, 1998, Komárek \& Anagnostidis 1998, 2005). The authority name of each species is cited in the text as described in 'Authors of Plant Names' (Brummitt \& Powell 1992), title of the books in citation is cited in accordance with Stafleu \& Cowan (1976, 1979, 1981, 1983, 1985, 1986, 1988) and supplements as described by Stafleu \& Mennege (1992, 1993, 1995, 1997, 1998, 2000), whereas Journals, Periodicals with Botanical content as described in "BotanicoPeriodicum-Huntianum", BPH-2 (Bridson 2004a, b).

\section{RESULTS}

Details of each new record of Cyanoprokaryotes of West Bengal from different water bodies of Maldah District in various blocks depicted in table 1 along with the geographical location.

Table 1. Cyanoprokaryotes new record from West Bengal in Maldah district.

\begin{tabular}{|c|c|c|c|c|}
\hline $\begin{array}{l}\text { S. } \\
\text { No. }\end{array}$ & $\begin{array}{l}\text { Name of the } \\
\text { Block }\end{array}$ & $\begin{array}{l}\text { Latitude, } \\
\text { Longitude }\end{array}$ & $\begin{array}{l}\text { Name of the } \\
\text { Water Bodies }\end{array}$ & Name of the species \\
\hline$\overline{1}$ & Ratua I & $\begin{array}{l}25^{\circ} 12^{\prime} 01.98^{\prime \prime} \mathrm{N} \\
87^{\circ} 56^{\prime} 56.26^{\prime \prime} \mathrm{E}\end{array}$ & $\begin{array}{l}\text { Bhadobartola } \\
\text { Pond }\end{array}$ & $\begin{array}{l}\text { Microcystis ichthyoblabe Kütz. } \\
\text { Microcystis panniformis Komárek }\end{array}$ \\
\hline \multirow[t]{2}{*}{2} & Ratua II & $\begin{array}{l}25^{\circ} 08^{\prime} 12.11^{\prime \prime N} \\
88^{\circ} 00^{\prime} 56.69^{\prime \prime} \mathrm{E}\end{array}$ & Barabilla Bil & $\begin{array}{l}\text { Pseudanabaena amphigranulata (Goor) Anagn. } \\
\text { Nostoc ellipsosporum var. violaceum C.B.Rao }\end{array}$ \\
\hline & & & Mahadhap Lake & $\begin{array}{l}\text { Pseudanabaena amphigranulata (Goor) Anagn. } \\
\text { Pseudanabaena limnetica (Lemmerm.) Komárek } \\
\text { Pseudanabaena redekei (Goor) B.A.Whitton }\end{array}$ \\
\hline \multirow[t]{3}{*}{3} & $\begin{array}{l}\text { Harishchandr } \\
\text { apur I }\end{array}$ & $\begin{array}{l}25^{\circ} 24^{\prime} 48.15^{\prime \prime N} \\
87^{\circ} 53^{\prime} 11.43^{\prime \prime} \mathrm{E}\end{array}$ & Damua Pond & $\begin{array}{l}\text { Oscillatoria crassa (C.B.Rao) Anagn. } \\
\text { Oscillatoria tenuis var. natans Gomont }\end{array}$ \\
\hline & & & Adhsoi Bil & Dactylococcopsis raphidioides Hansg. \\
\hline & & & Hazartakiya Bil & $\begin{array}{l}\text { Oscillatoria perornata f. attenuata Skuja } \\
\text { Anabaena inaequalis } \text { Bornet \& Flahault }\end{array}$ \\
\hline$\overline{4}$ & $\begin{array}{l}\text { Harishchandr } \\
\text { apur II }\end{array}$ & $\begin{array}{l}25^{\circ} 24^{\prime} 22.46^{\prime \prime} \mathrm{N} \\
87^{\circ} 52^{\prime} 00.23^{\prime \prime} \mathrm{E}\end{array}$ & Manna Bil & $\begin{array}{l}\text { Gloeocapsa nigrescens Nägeli } \\
\text { Aphanocapsa elachista } \text { var. conferta } \text { W.West \& } \\
\text { G.S.West }\end{array}$ \\
\hline$\overline{5}$ & Chanchal II & $\begin{array}{l}25^{\circ} 20^{\prime} 08.65^{\prime \prime} \mathrm{N} \\
88^{\circ} 01^{\prime} 09.92^{\prime \prime} \mathrm{E}\end{array}$ & Chakla Bil & $\begin{array}{l}\text { Komvophoron crassum (Vozžhenn.) Anagn. \& } \\
\text { Komárek }\end{array}$ \\
\hline$\overline{6}$ & Gazol & $\begin{array}{l}25^{\circ} 13^{\prime} 10.64^{\prime \prime} \mathrm{N} \\
88^{\circ} 10^{\prime} 13.19^{\prime \prime} \mathrm{E}\end{array}$ & $\begin{array}{l}\text { Kuchla Bil } \\
\text { Raikhan Dighi }\end{array}$ & $\begin{array}{l}\text { Jaaginema geitleri (Frémy) Anagn. \& Komárek } \\
\text { Heteroleibleinia mesotricha (Skuja) Anagn. \& } \\
\text { Komárek }\end{array}$ \\
\hline \multirow[t]{4}{*}{7} & Old Maldah & $25^{\circ} 00^{\prime} 31.46^{\prime \prime} \mathrm{N}$ & $\underline{\text { Sukan Dighi }}$ & Microcystis wesenbergii (Komárek) Komárek \\
\hline & & $88^{\circ} 09^{\prime} 29.21^{\prime \prime} \mathrm{E}$ & Paradhala Dighi & Synechocystis pevalekii Erceg. \\
\hline & & & Balutuli Bil & Pseudanabaena limnetica (Lemmerm.) Komárek \\
\hline & & & Nawabganj Bil & Anabaena vaginicola f. fertilissima B.N.Prasad \\
\hline \multirow[t]{4}{*}{8} & English Bazar & $\begin{array}{l}25^{\circ} 01^{\prime} 08.41^{\prime \prime} \mathrm{N} \\
88^{\circ} 14^{\prime} 10.98^{\prime \prime} \mathrm{E}\end{array}$ & Bara Sagar Dighi & $\begin{array}{l}\text { Microcystis novacekii (Komárek) Compère } \\
\text { Aphanocapsa rivularis (Carmich.) Rabenh. } \\
\text { Pseudanabaena dictyothalla (Skuja) Anagn. }\end{array}$ \\
\hline & & & Laxmipur Bil & Cyanosarcina burmensis (Skuja) Kovácik \\
\hline & & & Jorkuppa Pond & Pseudanabaena amphigranulata (Goor) Anagn. \\
\hline & & & Jalsukha Bil & $\begin{array}{l}\text { Oscillatoria angusta Koppe } \\
\text { Oscillatoria formosa f. loktakensis Brühl \& Biswas } \\
\text { Jaaginema geminatum (Schwabe ex Gomont) Anagn. } \\
\text { \& Komárek } \\
\text { Phormidium rimosum (Komárek) Anagn. \& Komárek }\end{array}$ \\
\hline
\end{tabular}




\section{Systematic Enumeration:}

Systematic studies carried out on Cyanoprokaryotes from diverse water bodies of Maldah district, West Bengal. Taxonomic enumeration of identified new record of Cyanoprokaryotes of West Bengal from Maldah district is described here along with their details.

\section{Chroococcales Wettst. \\ Chroococcaceae Nägeli \\ Cyanosarcina Kovácik}

Cyanosarcina burmensis (Skuja) Kovácik, Arch. Hydrobiol. Suppl. 80 (Algol. Stud. 50-53): 176. 1988. (Fig. 2A) Myxosarcina burmensis Skuja, Zur Süsswasseralgenflora Burmas 21, t. 1, f. 12. 1949.

Cells more or less angular with rounded corners, almost arranged in transverse and vertical series, pale bluegreen or olive-green, homogenous or finely granular; sheath very thin, mucilaginous, hyaline; colonies when young 4 cells.

Dimension: Cells 2.0-3.5 $\mu \mathrm{m}$ in diameter.

Distribution: Bil (Laxmipur).

\section{Dactylococcopsis Hansg.}

Dactylococcopsis raphidioides Hansg., Notarisia 590, 1888; Desikachary, Cyanophyta 158, t. 29, f. 1-2. 1959.

(Fig. 2B)

Cells light blue-green, spindle shaped, sigmoid or lunulately bent, single to a few together; species highly polymorphic.

Dimension: Cells 1.3-3.0 $\mu \mathrm{m}$ broad and 14.0-18.2 $\mu \mathrm{m}$ long.

Distribution: Bil (Adhsoi).

\section{Microcystaceae Elenkin Gloeocapsa Kütz.}

Gloeocapsa nigrescens Nägeli, in Rabenh., Alg. Sachs. 63, 629, 1857; Desikachary, Cyanophyta 117, t. 24, f. $15,17.1959$.

(Fig. 2C)

Cells spherical, surrounded by individual envelops, not lamellated, hyaline; cells uniting laterally, contents blue-green.

Dimension: Cells 3.0-6.0 $\mu \mathrm{m}$ in diameter.

Distribution: Bil (Manna).

Microcystis Lemmerm.

Microcystis ichthyoblabe (G.Kunze) Kütz., Phycol. general. 921, 1843; Komárek \& Anagn., Cyanoprokaryota Part 1: Chroococcales 19(1): 226, f. 297. 1998.

(Fig. 2D)

Granularia ichthyoblabe G.Kunze in E.Schmalz. Flora 6: 566. 1823.

Colony large, irregular, compact, without holes, mostly flattened, often form cell clusters or sub colonies in common mucilage, later on disintegrated in to small groups of aggregated cells; margins of colonies irregular, indistinct, diffuse, irregularly overlapping cells; cells spherical, densely homogeneously and evenly accumulated.

Dimension: Cells 2.0-3.7 $\mu \mathrm{m}$ in diameter.

Distribution: Pond (Bhadobartola).

Microcystis novacekii (Komárek) Compère, Bull. Jard. Bot. Natl. Belg. 44: 19, 1974; Komárek \& Anagn., Cyanoprokaryota Part 1: Chroococcales 19(1): 220, f. 302. 1998.

(Fig. 2E)

Diplocystis novacekii Komárek, in Komárek \& Ettl., Alg. Stud.: 63, t. 6, f. 1-4. 1958.

Colony almost spheroidal and slightly flattened, sometimes cells aggregated together; cells densely agglomerated in the centre of the colony, few solitary cells in enveloping mucilage.

Dimension: Cells 2.3-6.0 $\mu \mathrm{m}$ in diameter.

Distribution: Dighi (Bara Sagar).

Microcystis panniformis Komárek, Komárk.-Legn., C.L. Sant'Anna, M.T.P. Azevedo \& Senna, Crypto. Algol. 23: 165,f. 14-28. 2002; Komárek \& Anagn., Cyanoprokaryota Part1: Chroococcales 19(1): 226, f. 297. 1998. (Fig. 2F) 
Colony flat, irregular with small holes; margins of the colonies smooth or irregular; cells regularly densely and smoothly accumulated.

Dimension: Cells 2.5-4.9 $\mu \mathrm{m}$ in diameter.

Distribution: Pond (Bhadobartola).

Microcystis wesenbergii (Komárek) Komárek ex Komárek, in Kondrateva Cvetenie vody, Naukova Dumka

Kiev 32, 1968; Komárek \& Anagn., Cyanoprokaryota Part 1: Chroococcales 19(1): 232, f. 305.1998.

(Figs. 2G-K)

Diplocystis wesenbergii Komárek, Komárek \& Ettl., Alg. Stud.: 68, t. 7, f. 1-4. 1958.

Colony irregular, spheroidal to lobate or elongate with holes when old; mostly composed with connected spheroidal sub-colonies; cells sparsely to densely accumulated often near the surface of sub-colonies.

Dimension: Cells 4.0-8.6 $\mu \mathrm{m}$ in diameter.

Distribution: Dighi (Sukan).

\section{Nostocales Borzì \\ Nostocaceae Eichler \\ Anabaena Bory ex Bornet \& Flahault}

Anabaena inaequalis Bornet \& Flahault, Ann. Sci. Nat. Bot. ser. 7(7): 225, 231, 1886 (1888); N.D.Kamat, Hydrobiologia 22: 277, 1963.

(Fig. 2O)

Trichome almost straight or sometimes slightly twisted; cells short, barrel-shaped or truncate globose; heterocysts globose; spore not found in the sample.

Dimension: Cells 4.0-5.3 $\mu \mathrm{m}$ broad; heterocysts 5.0-6.8 $\mu \mathrm{m}$ broad.

Distribution: Bil (Hazartakiya).

Anabaena vaginicola f. fertilissima B.N.Prasad, J. Indian Bot. Soc. 31: 361, f. 14-17. 1952; Desikachary, Cyanophyta 401, t. 73, f. 3. 1959.

(Fig. 2P)

Trichome single or in a common mucilaginous sheath, blue-green; cells quadratic barrel-shaped, constricted at the cross-walls; heterocysts barrel-shaped slightly flattened; spore ellipsoidal, more than one or in the chain. Dimension: Trichome 4.8-5.7 $\mu \mathrm{m}$ broad and cells 3.2-5.4 $\mu \mathrm{m}$ long; heterocysts 5.8-6.8 $\mu \mathrm{m}$ broad and 5.0-6.0 $\mu \mathrm{m}$ long; spores 5.6-8.2 $\mu \mathrm{m}$ broad and 6.8-9.0 $\mu \mathrm{m}$ long.

Distribution: Bil (Nawabganj).

\section{Nostoc Vaucher ex Bornet \& Flahault}

Nostoc ellipsosporum var. violaceum C.B.Rao, Proc. Indian Acad. Sci., B. 6: 359, f. 4C, 1937; Desikachary,

Cyanophyta 383, t. 68, f. 4. 1959. ('violacea').

(Fig. 2Q)

Thallus gelatinous, irregularly expanded; filament flexuous, loosely entangled; trichome constricted at the cross-walls; cells cylindrical or almost quadrate; heterocysts almost spherical or cylindrical with the rounded flat end; spores ellipsoidal, almost spherical or cylindrical with the smooth outer wall.

Dimension: Trichome 3.2-3.6 $\mu \mathrm{m}$ broad and cells $2.5-7.9 \mu \mathrm{m}$ long; heterocysts $4.0-6.2 \mu \mathrm{m}$ broad and $4.8-7.8 \mu \mathrm{m}$ long; spores 5.0-7.0 $\mu \mathrm{m}$ broad and 5.6-12.0 $\mu \mathrm{m}$ long.

Distribution: Bil (Barabilla).

Oscillatoriales Schaffner

Gomontiellaceae Elenkin ex Geitler

Komvophoron Anagn. \& Komárek

Komvophoron crassum (Vozžhenn.) Anagn. \& Komárek, Arch. Hydrobiol., Suppl. (Algol. Stud. 50-53) 80: 373. 1988.

(Fig. 2R)

Pseudanabaena crassa Vozžhenn., Bot. Mater. Bot. Inst. Akad. Nauk. SSSR, Spor. Rast. 9: 73, f. 1: 2.1953.

Trichome curved sometimes more or less straight, short 8 to 80 celled, rarely more cells, distinctly constricted at the cross-walls; cells cylindrical; apical cell almost rounded.

Dimension: Trichome 4.1-4.9 $\mu \mathrm{m}$ broad and cells 4.4-5.6 $\mu \mathrm{m}$ long.

Distribution: Bil (Chakla).

\section{Oscillatoriaceae Kirchn.}


Oscillatoria Vaucher ex Gomont

Oscillatoria angusta Koppe, Arch. Hydrobiol. Planktonk. 14: 641, 1923; Desikachary, Cyanophyta 227, 1959.

(Fig. 2S)

Trichome solitary, blue-green, not constricted at the cross-walls; cells cylindrical, longer than broad; cell contents homogenous; apical cell almost cylindrical rounded.

Dimension: Trichome 1.0-1.8 $\mu \mathrm{m}$ broad and cells 5.0-7.5 $\mu \mathrm{m}$ long.

Distribution: Bil (Jalsukha).

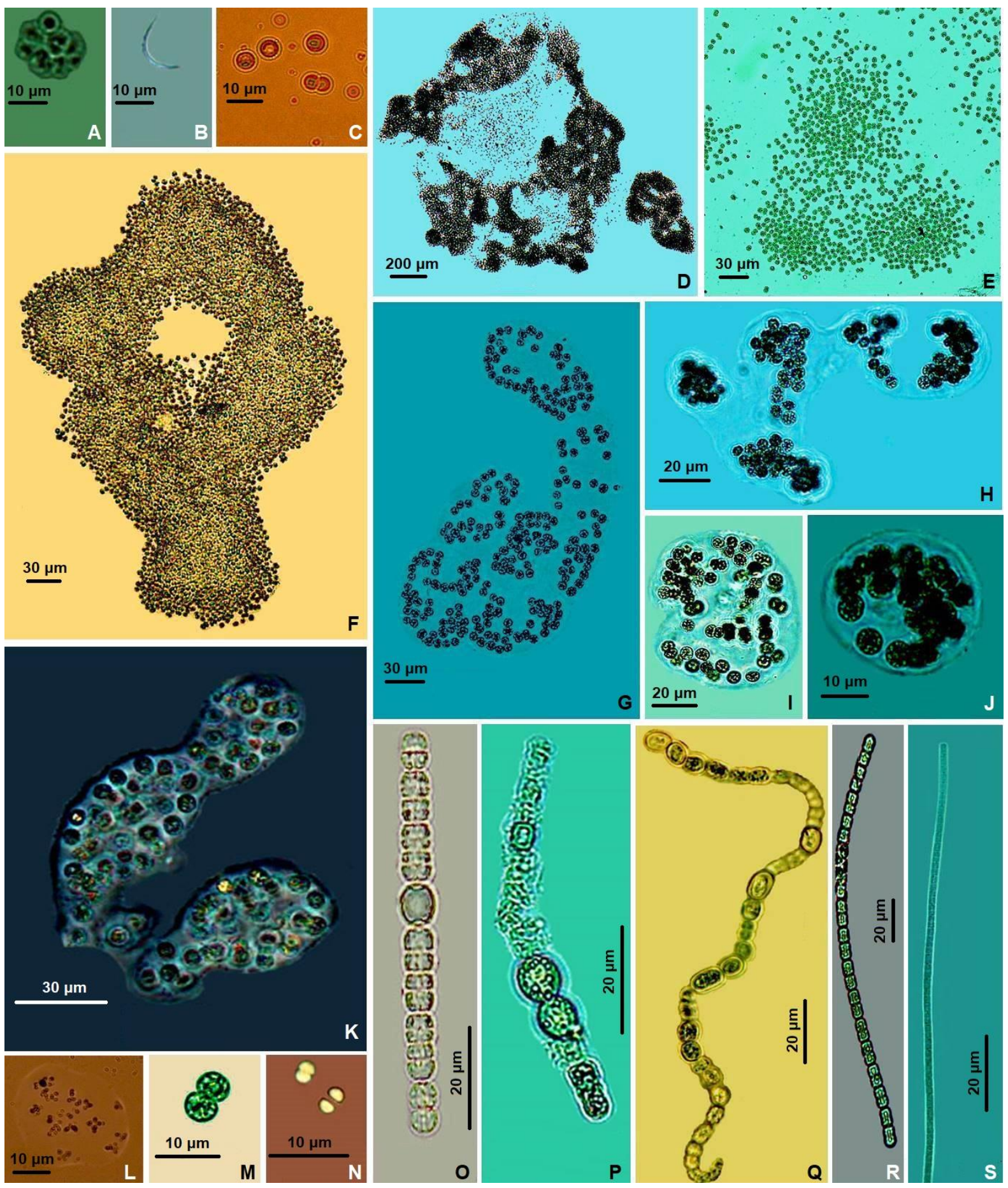

Figure 2. A, Cyanosarcina burmensis (Skuja) Kovácik; B, Dactylococcopsis raphidioides Hansg.; C, Gloeocapsa nigrescens Nägeli; D, Microcystis ichthyoblabe Kütz.; E, M. novacekii (Komárek) Compère; F, M. panniformis Komárek; G-K, M. wesenbergii (Komárek) Komárek; L, Aphanocapsa elachista var. conferta W.West \& G.S.West; M, A. rivularis (Carmich.) Rabenh.; N, Synechocystis pevalekii Erceg.; O, Anabaena inaequalis Bornet \& Flahault; P, Anabaena vaginicola f. fertilissima B.N.Prasad; Q, Nostoc ellipsosporum var. violaceum C.B.Rao; R, Komvophoron crassum (Vozžhenn.) Anagn. \& Komárek; S, Oscillatoria angusta Koppe.

www.tropicalplantresearch.com 
Oscillatoria formosa f. loktakensis Brühl \& Biswas, Mem. Asiat. Soc. Bengal 8: 264, t. 1, f. 5. 1926; Desikachary, Cyanophyta 233, t. 39, f. 4. 1959.

Trichome almost straight or slightly bent, flexible, slightly constricted at the cross-walls; end cell slightly attenuated; septa prominent.

Dimension: Trichome 4.0-6.0 $\mu \mathrm{m}$ broad and cells $2.2-3.0 \mu \mathrm{m}$ long.

Distribution: Bil (Jalsukha).

Oscillatoria crassa (C.B.Rao) Anagn., Preslia 73: 372, 2001.

(Fig. 3B)

Oscillatoria ornata var. crassa C.B.Rao, Proc. Indian Acad. Sci., B. 8: 165, f. 20. 1938.

Trichome straight and uniform in thickness, constricted at the cross-walls, granulated, dark blue-green in colour; cells shorter than broad; end cell convex, not capitate; calyptra absent.

Dimension: Trichome 12.0-15.0 $\mu \mathrm{m}$ broad and cells $2.8-5.2 \mu \mathrm{m}$ long.

Distribution: Pond (Damua).

Oscillatoria perornata f. attenuata Skuja, Nova Acta Regiae Soc. Sci. Upsal. ser. 4(14): 47, t. 8, f. 10. 1949;

Desikachary, Cyanophyta 205, t. 41, f. 7. 1959.

(Fig. 3C)

Trichome narrow, apices slightly attenuated and bent or curved, pale blue-green with or sometimes without gas-vacuoles; cells highly granular including septa.

Dimension: Trichome 9.3-11.8 $\mu \mathrm{m}$ broad and cells $2.8-5.8 \mu \mathrm{m}$ broad.

Distribution: Bil (Hazartakiya).

Oscillatoria tenuis var. natans Gomont, Ann. Sci. Nat. Bot. ser. 7(16): 221, f. 2-3. 1892; Prescott, Algae of the Western Great Lakes Area 491, t. 110, f. 11. 1982; S.Sharma \& M.L.Naik, Phykos 35: 140, 1996. (Fig. 3D)

Trichome straight, blue-green; cells broader than long; apical cell almost rounded.

Dimension: Trichome 5.2-8.5 $\mu \mathrm{m}$ broad and cells 3.4-4.2 $\mu \mathrm{m}$ long.

Distribution: Pond (Damua).

\section{Phormidium Kütz. ex Gomont}

Phormidium rimosum (Komárek) Anagn. \& Komárek, Arch. Hydrobiol., Suppl. (Algol. Stud. 50-53)80: 408, 1988; Komárek \& Anagn., Cyanoprokaryota Part 2: Oscillatoriales 19(2): 440, f. 641. 2005.

Lyngbya rimosa Komárek, Preslia 28: 374, f. 3 T - U \& f. 4.1956.

(Fig. 3E)

Thallus dark blue-green; filament short; sheath firm, attached to trichome, confluent within the colony; trichome cylindrical, not constricted or sometimes very slightly constricted at the cross-walls; not attenuated towards ends; cells usually broader than long, granulated; apical cell usually rounded; calyptra absent.

Dimension: Trichome 2.8-4.0 $\mu \mathrm{m}$ broad and cells 1.6-2.8 $\mu \mathrm{m}$ long.

Distribution: Bil (Jalsukha).

Synechococcales L.Hoffm., Komárek \& J.Kastovsky

Heteroleibleiniaceae (Komárek \& Anagn.) Komárek, J.Kastovsky, J.Mares \& J.R.Johans. Heteroleibleinia (Geitler) L.Hoffm.

Heteroleibleinia mesotricha (Skuja) Anagnostidis \& Komárek, Arch. Hydrobiol., Suppl. 80: 434, 1988. (Fig. 3F) Lyngbya mesotricha Skuja, Nova Acta Regiae Soc. Sci. Upsal. ser. 4, 14(15): 54, t. 9, f. 1-7. 1949.

Filament more or less curved, fixed to the substratum by the basal portion; sheath thin to moderately broad, firm, colourless; trichome end not attenuated, not constricted at the cross-walls, marked with one or two large granules on either side of cross-walls; cell contents pale blue-green, homogeneous; apical cell round or rounded conical; calyptra absent.

Dimension: Trichome 2.0-3.5 $\mu \mathrm{m}$ broad and cells 4.0-7.0 $\mu \mathrm{m}$ long.

Distribution: Dighi (Raikhan).

Pseudanabaenaceae Anagn. \& Komárek

Jaaginema Anagn. \& Komárek

Jaaginema geitleri (Frémy) Anagn. \& Komárek, Arch. Hydrobiol. Suppl. 80 (Algol. Stud. 50-53): 395.1988.

Oscillatoria geitleri Frémy, Arch. Bot. Mém. 3(2): 216, f. 185. 1930.

(Fig. 3G) 
Trichome single, long, blue-green, not constricted at the cross-walls; end erect, not attenuated, subcapitate; septa not granulated.

Dimension: Trichome 2.2-2.7 $\mu \mathrm{m}$ broad and cells 3.0-3.9 $\mu \mathrm{m}$ long.

Distribution: Bil (Kuchla).

Jaaginema geminatum (Schwabe ex Gomont) Anagnostidis \& Komárek, Arch. Hydrobiol. Suppl. 80 (Algol.

Stud. 50-53): 395. 1988.

(Fig. 3H)

Oscillatoria geminata Schwabe ex Gomont, Ann. Sci. Nat. Bot. ser. 7(16): 222, t. 7, f. 6. 1892.

Trichome curved or sometimes straight, blue-green, constricted at the cross-walls; not attenuated at the end; cells usually longer than broad; apical cell more or less rounded without calyptra.

Dimension: Trichome 2.3-3.3 $\mu \mathrm{m}$ broad and cells 2.2-5.6 $\mu \mathrm{m}$ long.

Distribution: Bil (Jalsukha).
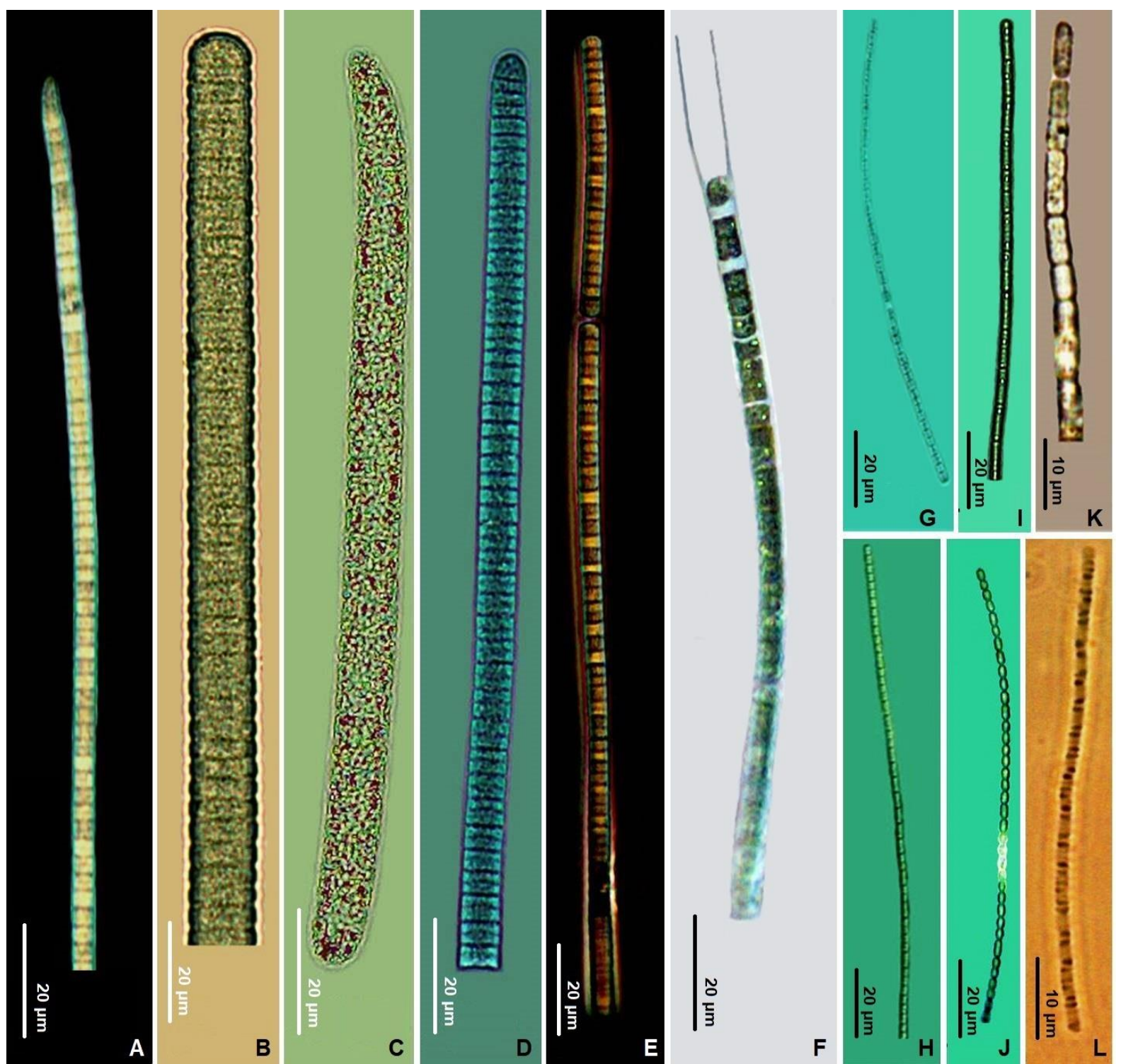

Figure 3. A, Oscillatoria formosa f. loktakensis Brühl \& Biswas; B, Oscillatoria crassa (C.B.Rao) Anagn.; C, O. perornata f. attenuata Skuja; D, O. tenuis var. natans Gomont; E, Phormidium rimosum (Komárek) Anagn. \& Komárek; F, Heteroleibleinia mesotricha (Skuja) Anagnostidis \& Komárek; G, Jaaginema geitleri (Frémy) Anagn. \& Komárek; H, J. geminatum (Schwabe ex Gomont) Anagnostidis \& Komárek; I, Pseudanabaena amphigranulata (Goor) Anagn.; J. $P$. dictyothalla (Skuja) Anagn.; K, P. limnetica (Lemmerm.) Komárek; L, P. redekei (Goor) B.A.Whitton.

\section{Pseudanabaena Lauterborn}

Pseudanabaena amphigranulata (Goor) Anagn., Preslia 73: 360. 2001.

(Fig. 3I)

Oscillatoria amphigranulata Goor, Recueil. Trav. Bot. Néerl. 15: 255, t. 2, f. 2. 1918. 
Trichome almost straight, constricted at the cross-walls with prominent granules; end not attenuated, not capitates; end cell rounded, calyptra absent.

Dimension: Trichome 1.2-2.0 $\mu \mathrm{m}$ broad and cells 1.8-5.6 $\mu \mathrm{m}$ long.

Distribution: Bil (Barabilla), Lake (Mahadhap) and Pond (Jorkuppa).

Pseudanabaena dictyothalla (Skuja) Anagn., Preslia 73: 360, 2001; Komárek \& Anagn., Cyanoprokaryota Part

2: Oscillatoriales 19(2): 80, f. 52. 2005.

Phormidium dictyothallum Skuja, Symb. Bot. Upsal. 9(3):51, t. 5, f. 8-11. 1948.

(Fig. 3J)

Trichome more or less straight or slightly bent; constricted at the cross-walls, not attenuated at the end; cells almost cylindrical, blue-green with rounded ends; cell contents homogenous; apical cell rounded; calyptra absent.

Dimension: Trichome 1.2-2.0 $\mu \mathrm{m}$ broad and cells 2.8-5.0 $\mu \mathrm{m}$ long.

Distribution: Dighi (Bara Sagar).

Pseudanabaena limnetica (Lemmerm.) Komárek, Sborn. Jihočesk. Muz. Ceských Budějovicich, Přir. Vedy 14: 162. 1974.

(Fig. 3K)

Oscillatoria limnetica Lemmerm., Ber. Duetsch. Bot. Ges. 18: 310, 1900.

Trichome pale blue-green, straight sometimes slightly bent, distinctly constricted at the cross-walls, not attenuated and capitates; end cell rounded; calyptra absent.

Dimension: Trichome 2.0-2.7 $\mu \mathrm{m}$ broad and cells 4.0-6.0 $\mu \mathrm{m}$ long.

Distribution: Bil (Balutuli) and Lake (Mahadhap).

Pseudanabaena redekei (Goor) B.A.Whitton, Cyanobacteria (Cyanophyta) 110, 2011.

Oscillatoria redekei Goor, Recueil. Trav. Bot. Néerl. 15: 258, t. 2, f. 3. 1918; Komárek \& Anagn., Cyanoprokaryota Part 2: Oscillatoriales 19(2): 440, f. 641. 2005.

Trichome solitary, straight or slightly curved, pale blue-green or yellow-green, not or slightly constricted at the cross-walls; not attenuated at the end, not capitate; cells usually longer than broad with two small or large polar aerotopes at the septa; apical cell almost rounded; calyptra absent.

Dimension: Trichome 1.4-2.5 $\mu \mathrm{m}$ broad and cells $2.5-6.0 \mu \mathrm{m}$ long.

Distribution: Jheel (Mahadhap).

\section{Merismopediaceae Elenkin \\ Aphanocapsa Nägeli}

Aphanocapsa elachista var. conferta W.West \& G.S.West, J. Linn. Soc., Bot. 40: 432, t. 19, f. 1. 1932; Desikachary, Cyanophyta 133, t. 22, f. 10. 1959.

(Fig. 2L)

Colony ellipsoidal or almost oval or spherical; mucilage colourless; cells more or less closely arranged.

Dimension: Cells 1.4-2.0 $\mu \mathrm{m}$ in diameter and colony 56.0-71.0 $\mu \mathrm{m}$ in diameter.

Distribution: Bil (Manna).

Aphanocapsa rivularis (Carmich.) Rabenh., Fl. eur. alg. 11: 49, 1865. Prescott, Algae of the Western Great Lakes Area 454, t. 101, f. 17. 1982.

(Fig. 2M)

Palmella rivularis Carmich., in Hook., Brit. Fl. 2(1): 397, 1833.

Cells globose, with bright blue-green granular contents, usually solitary or in pairs, scattered within the colonial mucilage envelope.

Dimension: Cells 5.0-6.0 $\mu \mathrm{m}$ in diameter.

Distribution: Dighi (Bara Sagar).

\section{Synechococcaceae Komárek \& Anagno. Synechocystis Sauv.}

Synechocystis pevalekii Erceg., Acta Bot. Inst. Bot. Univ. Zagreb 1: 77, t. 1, f. 8. 1925; Desikachary, Cyanophyta 145, t. 25, f. 11. 1959.

(Fig. 2N)

Cells spherical, two together or sometimes single, generally without visible gelatinous matrix.

Dimension: Cells 2.2-3.5 $\mu \mathrm{m}$ in diameter.

Distribution: Dighi (Paradhala). 


\section{DISCUSSION AND CONCLUSION}

Altogether 27 species (including 03 variety and 03 forms) have been reported from 18 water bodies of Maldah District, West Bengal. Out of 13 blocks of Maldah District Cynoprokaryotes were recorded from 03 Ponds, 10 Bils, 01 Lake and 04 Dighi of 08 blocks namely Ratua I, Ratua II, Harishchandrapur I, Harishchandrapur II, Chanchal II, Gazol, Old Maldah and English Bazar. Blockwise maximum 09 new records were observed from different water bodies of English Bazar viz. Aphanocapsa rivularis (Carmich.) Rabenh., Cyanosarcina burmensis (Skuja) Kovácik, Jaaginema geminatum (Schwabe ex Gomont) Anagn. \& Komárek, Microcystis novacekii (Komárek) Compère, Oscillatoria angusta Koppe, Oscillatoria formosa f. loktakensis Brühl \& Biswas, Phormidium rimosum (Komárek) Anagn. \& Komárek, Pseudanabaena amphigranulata (Goor) Anagn. and Pseudanabaena dictyothalla (Skuja) Anagn. and again maximum 04 species observed only from Jalsukha Bil of English Bazar followed 02 species from each water bodies of block Gazol, Harishchandrapur I, Harishchandrapur II, Old Maldah, Ratua I and Ratua II. However, single species observed from Chakla Bil of block Chachal II i.e. Komvophoron crassum (Vozžhenn.) Anagn. \& Komárek. It was observed that some of the species have been observed in more than one water bodies like Pseudanabaena amphigranulata (Goor) Anagn. from Barabilla Bil, Mahadhap Lake and Jorkuppa Pond and Pseudanabaena limnetica (Lemmerm.) Komáre from, Balutuli Bil and Mahadhap Lake. From these studies, one can easily explain the ecological preferences of various species of Cyanoprokaryota. These taxonomic studies may be used in experimental as well as ecological studies. This study will fill the gap in our existing knowledge of the biodiversity of the Cyanoprokaryotes of this area. A new distributional record recorded from West Bengal of Maldah District, which we expect to be utilized by experimental and evolutionary researchers worldwide. Further Molecular studies in combination with morphological studies will provide new insights into the real diversity of Cyanoprokaryota and their biogeographical distribution in this environment. However, many more studies are needed to unravel the enormous diversity of Cyanoprokaryota and to better define their bio-geographical patterns.

\section{ACKNOWLEDGEMENTS}

The author is thankful to the Director, Botanical India, Ministry of Environment Forest \& Climate Change, Government of India, Kolkata for proving the necessary laboratory facilities for completion of this work.

\section{REFERENCES}

Anand N (1989) Hand Book of Blue-Green Algae. Bishen Singh Mahendra Pal Singh, Dehradun, India, pp. 79. Anand N (1998) Indian fresh water microalgae. Bishen Singh Mahendra Pal Singh, Dehradun, India, pp. 94.

BAES (2004) District Statistical Handbook, Maldah. Bureau of Applied Economics and Statistic, Government of West Bengal, Kolkata, pp. 174.

Bajpai O, Mishra S, Mohan N, Mohan J \& Gupta RK (2013) Phyco chemical characteristics of Lakhna Devi temple water tank, Lakhna, Bakewar, Etawah, U.P. with reference to Cynobacterial Diversity. International Journal of Environment 1(1): 20-28.

Banerjee A \& Santra SC (2001) Phytoplanktons of the rivers of Indian Sunderban mangrove estuary. Indian Biologist 33(1): 67-71.

Banerjee G (1997) Check list of algae in domestic sewage of Calcutta. Environment and Ecology 15(3): 548552.

Banerjee G (1998) Survey of algae in habitats influenced by agro-chemical effluents and domestic sewage. Environment and Ecology 16(1): 132-134.

Biswas K (1925) Road slimes of Calcutta. Journal of the Department of Science of Calcutta University 7: 1-10.

Biswas K (1926) Flora of Salt Lakes, Calcutta. Journal of the Department of Science of Calcutta University 8: $1-47$.

Bridson GDR (2004a) Botanico-Periodicum-Huntianum (BPH - 2). $2^{\text {nd }}$ Ed., 1(A - M): 1-819 pp.

Bridson GDR (2004b) Botanico-Periodicum-Huntianum (BPH - 2). $2^{\text {nd }}$ Ed., 2(N - Z): 821-1470 pp.

Brummitt RK \& Powell CE (1992) Authors of Plant Names. Royal Botanic Gardens, Kew, pp. 732.

Chatterjee M \& Chatterjee SP (1983) Nitrogen fixing Cyanobacteria from the paddy field soils of Burdwan District. Phykos 22: 64-66.

Chatterjee M \& Choudhury A (1980) Algal flora of the water reservoirs of Golapbag, Burdwan, West Bengal. Phykos 19(1): 67-69.

Desikachary TV (1959) Cyanophyta. Indian Council of Agricultural Research, New Delhi, pp. 686. 
Geitler L (1932) Cyanophyceae. In: Rabenhorst, L. (ed.), Kryptogammenflora von Deutschland, Osterreich, under de Sweitz. Akad. Verlagsges, Leipzing 14: pp 1196.

Gupta D \& Sen C (1978) Observation on blue-green algae of paddy field soils of Gangetic West Bengal. I. Burdwan District. Indian agriculturist 31(3): 221-225.

Gupta D \& Sen C (1987) Some Cyanophyceae from Gangetic delta of West Bengal - I. Hooghly District - A taxonomic enumeration. Journal of Economic and Taxonomic Botany 10(2): 381-385.

Halder N (2015) Phytoconstituents composition and in vitro antibacterial activity of a blue green alga Anabaena variabilis Kütz. ex Born. et Flah. Tropical Plant Research 2(3): 288-291.

Jitendra P \& Anand VK (2016) Eight new records of fresh water filamentous algae (Oedogonium Link) from India. Tropical Plant Research 3(1): 33-39.

Komárek J \& Anagnostidis K (1998) Cyanoprokaryota 1. Teil: Chroococcales. In: Ettl H, Gärtner G, Heynig H \& Mollenhauer D (eds) Süsswasserflora von Mitteleuropa, Gustav Fischer, Jena-Stuttgart-Lübeck-Ulm. 19(1): pp. 548.

Komárek J \& Anagnostidis K (2005) Cyanoprokaryota $2^{\text {nd }}$ Part : Oscillatoriales. In: Büdel B, Krienitz L, Gärtner G \& Schagerl M (eds) Süsswasserflora von Mitteleuropa, Elsevier / Spektrum, Heidelberg 19(2): pp. 759.

Maity H \& Santra SC (1985) Blue-green algal flora of 24 Paraganas, West Bengal (India). Phykos 24: 46-51.

Malathi T, Babu MR, Mounika T, Snehalatha D \& Rao BD (2014) Screening of cyanobacterial strains for antibacterial activity. Phycological Society of India 44(2): 6-11.

Mitra S \& Gupta D (1994) The genus Nostoc Vaucher and Anabaena Bory from Greater Calcutta. Journal of the National Botanical Society 48: 77-80.

Mohan J, Narain S, Kumar H \& Mohan N (2007) Diversity of blue green algae in Allen Forest Lake, Zoological Park, Kanpur, mixing with campus sewage. Indian Hydrobiology 10(1): 123-127.

Mukherjee D (1986) Seasonal variation of blue-green algae on the surface of uncultivated upland soils of Bankhura District (West Bengal). Geobios New Reports 5(1): 39-41.

Mukhopadhyay A \& Chatterjee P (1981) A check-list of blue-green algae from the paddy fields of 24 Parganas and Howrah District of West Bengal - I. Phykos 20(1\&2): 81-84.

Naskar NM, Naskar KR \& Talai S (2008) Occurrence of six species of Spirulina Turpin em Gardner from the coastal wetlands of North 24 Parganas District, West Bengal, India, Seshaiyana ENVIS on estuaries, mangroves, coral reefs and lagoons 16(1): 4-5.

Pal TK \& Santra SC (1982) Contribution to the Cyanophyceae of Murshidabad. Phykos 21: 150-152.

Pal TK \& Santra SC (1984) New addition to the algal flora of Murshidabad, West Bengal. Phykos 23(1 \& 2): 139-141.

Pal TK, Adhya TK \& Santra SC (1986) Algal flora of Murshidabad District, West Bengal I. A survey from Berhampore and adjoining areas. Bulletin of the Botanical Society of Bengal 40: 33-43.

Pal UC \& Santra SC (1985) Algal flora of Midnapur, West Bengal (India) - 1, Cyanophyceae. Phykos 24: 1217.

Pal UC, Naskar KR \& Santra SC (1988) A check list of algal flora of Sundarban delta of West Bengal, India. Phykos 27: 48-53.

Patel AK, Suseela MR, Singh M \& Nayaka S (2017) Change in physico-chemical character of nutrient media and carpet effluent in presence of six algae monocultures and their consortia. Tropical Plant Research 4(2): 192-202.

Prescott GW (1982) Algae of the Western Great Lakes Area. Otto Koeltz Science Publishers, W. Germany. pp. 977.

Sabata BC \& Nayer MP (1992) Taxonomy of algae of river Hooghly. Bulletin of the Botanical Survey of India 34(1-4): 194-204.

Santra SC (1987) Air borne algae of Calcutta Metropolis. Phykos 26: 71-74.

Sen C \& Gupta D (1993) Some Cyanophycean forms Gangetic delta of West Bengal II. Howrah District - A taxonomic enumeration. Journal of Economic and Taxonomic Botany 17(2): 312-314.

Sen CR (2006) Some Cyanophyceae from Greater Calcutta - I : A taxonomic enumeration. Journal of Economic and Taxonomic Botany 30(1): 885-890.

Sikdar J \& Keshri JP 2014 The Genus Oscillatoria Vaucher (Oscillatoriales: Cyanoprokaryota) in West Bengal, India. International Journal of Current Research and Review 6(21): 47-59.

Singh BV, Choudhary KK, Dhar DW \& Singh PK (2001) Occurrence of some Nostocales from 24 Paraganas, www.tropicalplantresearch.com 
West Bengal. Phykos 40(1 \& 2): 83-87.

Sinha JP \& Mukherjee D (1984) Blue-green algae from the paddy fields of Bankura District of West Bengal III. Phykos 23(1\&2): 142-143.

Sridhar R, Thangaradjou T, Kumar SS \& Kannan L (2006) Water quality and phytoplankton characteristics in the Palk Bay, South-East cost of India. Journal of Environmental Biology 27: 561-566.

Srivastava N, Suseela MR \& Toppo K (2014) Fresh water cyanobacteria of Sai River near Lucknow, Uttar Pradesh. Tropical Plant Research 1(2): 11-16.

Stafleu FA \& Cowan RS (1976) Taxonomic literature. Bohn, Scheltema \& Holkema, Utrecht 1(A-G): pp. 1134.

Stafleu FA \& Cowan RS (1979) Taxonomic literature. Bohn, Scheltema \& Holkema, Utrecht, dr. W. Junk b. v., Publishers, The Hague 2(H - Le): pp. 990.

Stafleu FA \& Cowan RS (1981) Taxonomic literature. Bohn, Scheltema \& Holkema, Utrecht, dr. W. Junk b. v., Publishers, The Hague 3(Lh - O): pp. 980.

Stafleu FA \& Cowan RS (1985) Taxonomic literature. Bohn, Scheltema \& Holkema, Utrecht, dr. W. Junk b. v., Publishers, The Hague 5(Sal - Ste): pp. 1066.

Stafleu FA \& Cowan RS (1986) Taxonomic literature. Bohn, Scheltema \& Holkema, Utrecht, dr. W. Junk b. v., Publishers, The Hague/Boston 6(Sti - Vuy): pp. 926.

Stafleu FA \& Cowan RS (1988) Taxonomic literature. Bohn, Scheltema \& Holkema, Utrecht/Antwerpen, dr. W. Junk b. v., Publishers, The Hague/Boston 7(W - Z): pp. 653.

Stafleu FA \& Mennege EA (1992) Taxonomic literature. Koeltz Scientific Books Königstein, Germany, Suppl. 1(A - Ba): pp. 442.

Stafleu FA \& Mennege EA (1993) Taxonomic literature. Koeltz Scientific Books Königstein, Germany, Suppl. 2(Be - Bo): pp. 464.

Stafleu FA \& Mennege EA (1995) Taxonomic literature. Königstein, Germany, Suppl. 3(Br - Ca): pp. 550.

Stafleu FA \& Mennege EA (1997) Taxonomic literature. Koeltz Scientific Books, Königstein, Germany, Suppl. 4(Ce-Cz): pp. 614.

Stafleu FA \& Mennege EA (1998) Taxonomic literature. Koeltz Scientific Books, Königstein, Germany, Suppl. 5(Da - Di): pp. 432.

Stafleu FA \& Mennege EA (2000) Taxonomic literature. Koeltz Scientific Books, Königstein, Germany, Suppl. 6(Do - E): pp. 518.

Stafleu FA \& Cowan RS (1983) Taxonomic literature. Bohn, Scheltema \& Holkema, Utrecht/Antwerpen, dr. W. Junk b. v., Publishers, The Hague/Boston 4(P - Sak): pp. 1188.

Tiffany LH \& Britton ME (1952) The Algae of Illinois. The University of Chicago Press, Chicago, Cambridge University Press, London, pp. 397. 